\title{
YOUNG MEASURES, CARTESIAN MAPS, AND POLYCONVEXITY
}

\author{
Patrick Bernard ANd Ugo Bessi
}

\begin{abstract}
We consider the variational problem consisting of minimizing a polyconvex integrand for maps between manifolds. We offer a simple and direct proof of the existence of a minimizing map. The proof is based on Young measures.
\end{abstract}

\section{Introduction}

Let $\bar{N}$ be a compact Riemannian manifold with boundary of dimension $n$ and $M$ be a complete Riemannian manifold of dimension $m$. We denote by $N$ the interior of $\bar{N}$, and by $\mathrm{d} t$ the non-negative Borel measure on $N$ associated with the metric. It can be defined as the $n$-Hausdorff measure associated with the Riemannian distance, and it exists also if $N$ is not orientable. This measure is also characterized by the fact that

$$
\int_{D} \mathrm{~d} t=\left|\int_{D} \Omega\right|
$$

for each embedded disk $D \subset M$, where $\Omega$ is any of the two unitary volume forms on $D$. We set $p=\min \{m, n\}$. We want to discuss the classical question of minimizing the quantity

$$
\int_{N} L\left(t, u(t), \mathrm{d} u_{t}\right) \mathrm{d} t
$$

on appropriate classes of maps $u: N \longrightarrow M$. We recall some terminology about Lagrangians:

Convexity: Given $k \in\{1, \ldots, \min (m, n)\}$, we say that the integrand $L(t, x, v)$ is $k$-convex if it can be written in the form

$$
L(t, x, v)=\mathrm{L}\left(t, x, v, \wedge_{2} v, \ldots, \wedge_{k} v\right)=\mathrm{L}_{t, x}\left(v, \wedge_{2} v, \ldots, \wedge_{k} v\right)
$$

with a Borel measurable function $\mathrm{L}$ such that

$\left.\mathrm{L}_{t, x}: \mathcal{L}\left(T_{t} N, T_{x} M\right) \times \mathcal{L}\left(\wedge_{2} T_{t} N, \wedge_{2} T_{x} M\right) \times \cdots \times \mathcal{L}\left(\wedge_{k} T_{t} N, \wedge_{k} T_{x} M\right)\right) \rightarrow \mathbb{R} \cup\{+\infty\}$

Received May 26, 2008.

2000 Mathematics Subject Classification. 49Q20, 49J45.

Key words and phrases. calculus of variations, polyconvexity, gradient measures.

(C)2010 The Korean Mathematical Society 
is convex for each $t$ and $x$. When $k=1$, this just says that $L$ is convex in $v$; when $k=p$, this hypothesis is usually called polyconvexity. Let us explain the notations. We denote by $\mathcal{L}(E, F)$ the set of linear maps between $E$ and $F$. The space $\wedge_{i} E$ is the vector space $E \wedge E \wedge \cdots \wedge E$ generated by elements of the form $e_{1} \wedge e_{2} \wedge \cdots \wedge e_{i}, e_{j} \in E$. Given $v \in \mathcal{L}(E, F)$, we denote by $\wedge_{i} v \in \mathcal{L}\left(\wedge_{i} E, \wedge_{i} F\right)$ the linear map such that

$$
\wedge_{i} v\left(e_{1} \wedge \cdots \wedge e_{i}\right)=v\left(e_{1}\right) \wedge v\left(e_{2}\right) \wedge \cdots \wedge v\left(e_{i}\right) .
$$

If $E$ has dimension $n$ and $F$ has dimension $m$, the dimension of $\wedge_{i} E$ is $C_{n}^{i}$ (binomial coefficient) and $\wedge_{i} v$ can be represented by a $C_{n}^{i} \times C_{m}^{i}$ matrix whose coefficients are the determinants of all $i \times i$ sub-matrices that can be extracted from the matrix representing $v$. Let us denote by $J^{1}(N, M)$ the manifold of 1-jets of maps from $N$ to $M$. If $N$ is an open subset of $\mathbb{R}^{n}$ and $M=\mathbb{R}^{m}$, then

$$
J^{1}(N, M)=N \times M \times \mathcal{L}\left(\mathbb{R}^{n}, \mathbb{R}^{m}\right) .
$$

Regularity: We say that $L: J^{1}(N, M) \longrightarrow \mathbb{R} \cup\{+\infty\}$ is a normal integrand if it is a Borel measurable function and if, for almost all $t \in N$, the function $(x, v) \longmapsto L(t, x, v)$ is lower semi-continuous.

Coercivity: We say that $L$ is $k$-superlinear if there exists a superlinear function $l:[0, \infty) \longrightarrow \mathbb{R}$ such that

$$
L(t, x, v) \geqslant l\left(\|v\|+\left\|\wedge_{2} v\right\|+\cdots+\left\|\wedge_{k} v\right\|\right)
$$

for all $(t, x, v) \in J^{1}(N, M)$.

Our goal is to explain a compact and simple approach to this kind of problems. We do not present real novelties, and much of the techniques we will use can be found in [14] or $[12,13]$. Yet we believe it is not useless to present the short path to Theorem 1 that follows. This work started with an attempt to extend the methods of [7] to higher dimension.

We will define, by studying relevant sets of Young measures, $\operatorname{sets~}_{\operatorname{cart}}(N, M)$ of maps $u: N \longrightarrow M$ such that

$$
W^{1, n}(N, M) \subset \operatorname{cart}_{\min \{n, m\}}(N, M) \subset \operatorname{cart}_{k}(N, M) \subset \operatorname{cart}_{1}(N, M)=W^{1,1}(N, M) .
$$

In the case $k=\min \{n, m\}$ the set that we denote $\operatorname{cart}_{k}(N, M)$ is similar to the set denoted $\operatorname{cart}^{1}(N, M)$ in $[12,13]$, but our presentation is quite different.

In order to state appropriately a variational problem, it is useful to specify boundary conditions. We assume that the boundary $\partial N$ of $\bar{N}$ is not empty and we fix a map $u_{0} \in \operatorname{cart}_{k}(N, M)$. We denote by $\operatorname{cart}_{k}\left(N, M ; u_{0}\right)$ the set of maps $u$ in $\operatorname{cart}_{k}(N, M)$ such that the trace of $u$ in $L^{1}(\partial N, M)$ is equal to the trace of $u_{0}$. These traces are well defined (at least in the case where $M$ is a Euclidean space) because $\operatorname{cart}_{k}(N, M) \subset W^{1,1}(N, M)$, and each element of $W^{1,1}(N, M)$ has a unique boundary trace in $L^{1}$ (see for instance [11] for the definition); we recall that the integration by parts formula holds for this trace. In the case

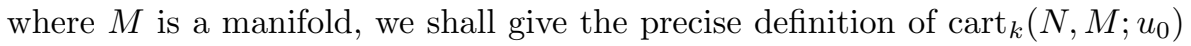


in Section 3. Our goal is to provide a short and direct proof of the following result:

Theorem 1. Let $k \in\{1, \ldots, \min (m, n)\}$ be given and let $L: J^{1}(N, M) \longrightarrow \mathbb{R} \cup$ $\{+\infty\}$ be a $k$-convex and $k$-superlinear normal integrand. Let $u_{0} \in \operatorname{cart}_{k}(N, M)$ be given, such that

$$
\int_{N} L\left(t, u_{0}(t), \mathrm{d} u_{0}(t)\right) \mathrm{d} t<\infty
$$

Then there exists a map $u \in \operatorname{cart}_{k}\left(N, M ; u_{0}\right)$ which minimizes the integral

$$
\int_{N} L(t, u(t), \mathrm{d} u(t)) \mathrm{d} t
$$

in $\operatorname{cart}_{k}\left(N, M ; u_{0}\right)$.

Note that, in general, we may have

$$
\inf _{u \in W^{1,1}\left(N, M ; u_{0}\right)} \int_{N} L\left(t, u(t), \mathrm{d} u_{t}\right) \mathrm{d} t<\min _{u \in \operatorname{cart}_{k}\left(N, M ; u_{0}\right)} \int_{N} L\left(t, u(t), \mathrm{d} u_{t}\right) d t
$$

and, even if $u_{0}$ is smooth,

$$
\min _{u \in \operatorname{cart}_{k}\left(N, M ; u_{0}\right)} \int_{N} L\left(t, u(t), \mathrm{d} u_{t}\right) \mathrm{d} t<\inf _{u \in C^{1}\left(N, M ; u_{0}\right)} \int_{N} L\left(t, u(t), \mathrm{d} u_{t}\right) \mathrm{d} t .
$$

The paper [4] contains an example in which $N$ has dimension 1 and the minimum on $W^{1,1}\left(N, \mathbb{R}^{n}\right)=\operatorname{cart}_{1}\left(N, \mathbb{R}^{n} ; u_{0}\right)$ is smaller than the infimum on $C^{1}$. As for the other strict inequality, we are going to see an example in Section 4.2.

Theorem 1 is a slight extension on the seminal results of Ball [3]. Compared to this work and to classical papers on polyconvexity, our proof works under slightly weaker coercivity. Our theorem reduces to the famous Tonelli Theorem in the case $n=1$, and to the famous De Giorgi Theorem in the case $m=$ 1. Several extensions are known, which go much beyond what we plan to expose. First, the kind of convexity hypothesis can be relaxed to the so-called quasi-convexity, but then one has to add more stringent growth conditions, see $[10,14,9]$. Second, one can, in certain circumstances, relax the coercivity condition to the case when $L$ has only linear growth, by using cartesian currents and functions of bounded variations. Excellent surveys of these methods are in $[12,13,9]$.

Our approach is based on Young measures, also called parametrized measures. A survey on the use of Young measure is the book [14]. Many of our techniques are adapted from this book. In Section 2, we define the various sets of Young measures that are useful, in particular the set of generalized maps, on which it is appropriate to relax the variational problem. We study the structure of generalized maps and conclude that minimizing generalized maps correspond to minimizing maps. In Section 3, we prove a compactness results under boundary conditions. In Section 4, we briefly expose how the various tools exposed in Sections 2 and 3 lead to a proof of Theorem 1. We also collect 
various related remarks. Finally, we recall in the Appendix some relevant facts on the topology of some spaces of measures.

We end this introductory section collecting some notation and material on $n$-vectors and $n$-forms we shall need in the following.

\subsection{Some algebra}

Let $E$ and $F$ be two Euclidean vector spaces of dimension $n$ and $m$. It will be convenient to set $p:=\min (m, n)$. We denote by $\mathcal{L}(E, F)$ the set of linear maps between $E$ and $F$. Recall that the vector space $\wedge_{l} E$ is endowed with a natural inner product (which is induced from the inner product of $E$ ). This inner product can be characterized by the property that

$$
\left\langle v_{1} \wedge \cdots \wedge v_{l}, w_{1} \wedge \cdots \wedge w_{l}\right\rangle=\operatorname{det}(G),
$$

where $G \in M^{l, l}(\mathbb{R})$ is the Gram matrix $G_{i, j}=\left\langle v_{i}, w_{j}\right\rangle$ and $\operatorname{det} G$ is the $\operatorname{deter-}$ minant of $G$. Note that

$$
\left\|v_{1} \wedge \cdots \wedge v_{l}\right\|:=\left\langle v_{1} \wedge \cdots \wedge v_{l}, v_{1} \wedge \cdots \wedge v_{l}\right\rangle^{1 / 2}=1
$$

if $\left(v_{1}, \ldots, v_{l}\right)$ is orthonormal in $E$. Given $a \in \mathcal{L}(E, F)$, we denote by $\|a\|:=$ $\sup _{\|x\|_{E} \leqslant 1}\|a(x)\|_{F}$ its norm and by $\wedge_{l} a \in \mathcal{L}\left(\wedge_{l} E, \wedge_{l} F\right)$ the unique linear map which satisfies

$$
\wedge_{k} a\left(v_{1} \wedge v_{2} \cdots \wedge v_{l}\right)=a\left(v_{1}\right) \wedge a\left(v_{2}\right) \wedge \cdots \wedge a\left(v_{l}\right)
$$

for each $v_{1}, \ldots, v_{l}$ in $E$. The map $\wedge_{l} a$ is called the $l$-adjoint of $a$. In coordinates, this map is represented by a matrix whose coefficients are the $l$-minors of $a$.

If $\omega$ is a $k$-form on $E$ and $U$ is an $l$-vector, $l \leqslant k$, we denote by $i_{U} \omega$ the $(k-l)$-form defined by

$$
i_{U} \omega \cdot v=\omega \cdot(U \wedge v)
$$

for any $(k-l)$-vector $v$.

Lemma 1. Let $\Omega$ be a volume form on $E$, let $\lambda$ be the unique $n$-vector on $E$ such that $\Omega \cdot \lambda=1$, let $U$ be a k-vector on $E$ and let $a \in \mathcal{L}(E, F)$. Then, for any $k$-form $\chi$ on $F$, we have

$$
\left(\left(i_{U} \Omega\right) \wedge \chi\right) \circ \wedge_{n}(I d \oplus a) \cdot \lambda=(-1)^{k(n-k)} \chi \circ \wedge_{k} a \cdot U
$$

where $I d \oplus a: E \longrightarrow E \oplus F$ is the map $v \longmapsto(v, a(v))$.

Proof. We make a proof in coordinates. Let $\left(e_{1}, \ldots, e_{n}\right)$ be a base of $E$ such that $\lambda=e_{1} \wedge e_{2} \wedge \cdots \wedge e_{n}$. If $e_{j}^{*}$ is the dual base of $E^{*}$, then we have $\Omega=e_{1}^{*} \wedge \cdots \wedge e_{n}^{*}$. If $I$ is a subset of $\{1, \ldots, n\}$, we denote by $e_{I}$ the product $e_{\alpha_{1}} \wedge \cdots \wedge e_{\alpha_{i}}$, where $i$ is the cardinal of $I$, and $\alpha_{j}, 1 \leqslant j \leqslant i$ are the elements of $I$ in increasing order. We denote by $\sigma(I)$ the sign such that $e_{I} \wedge e_{I^{c}}=\sigma(I) \lambda$, where $I^{c}$ is the complement of $I$. Note that $\Omega=\sigma(I) e_{I}^{*} \wedge e_{I^{c}}^{*}$, so that $i_{e_{I}} \Omega=\sigma(I) e_{I^{c}}^{*}$. Note that 
$\sigma\left(I^{c}\right)=(-1)^{k(n-k)} \sigma(I)$. It is sufficient to prove the lemma for $U=\sigma\left(J^{c}\right) e_{J^{c}}$, where $J$ has cardinal $n-k$, in which case $i_{U} \Omega=e_{J}^{*}$. We have

$$
\wedge_{n}(I d \oplus a) \cdot \lambda=\sum_{I} \sigma(I) e_{I} \wedge\left(\wedge_{\left|I^{c}\right|} a \cdot e_{I^{c}}\right),
$$

where the sum is taken on all subsets $I$ of $\{1, \ldots, n\}$ and $|I|$ is the cardinal of I. We get

$$
\begin{aligned}
& \left(\left(i_{U} \Omega\right) \wedge \chi\right) \circ \wedge_{n}(I d \oplus a) \cdot \lambda=\left(e_{J}^{*} \wedge \chi\right) \circ \wedge_{n}(I d \oplus a) \cdot \lambda \\
= & \sigma(J) \chi \circ \wedge_{k} a \cdot e_{J^{c}}=(-1)^{k(n-k)} \chi \circ \wedge_{k} a \cdot U .
\end{aligned}
$$

\section{Generalized maps}

Let us first recall the definitions of the Sobolev space $W^{1, q}(N, M)$. We say that $u$ belongs to this space if there exists a $v(t) \in \mathcal{L}\left(T_{t} N, T_{u(t)} M\right)$, depending measurably on $t$, such that

$$
\int_{N}\|v(t)\|_{t, u(t)}^{q} \mathrm{~d} t<\infty
$$

and

$$
\mathrm{d}(\chi \circ u)_{t}=\mathrm{d} \chi_{u(t)} \circ v(t)
$$

in the sense of distributions for all bounded smooth functions $\chi: M \longrightarrow \mathbb{R}$ with bounded derivative. This can be written intrinsically on the manifold $N$ by requiring that, for each smooth vectorfield $U(t)$ on $N$ compactly supported in the interior of $N$, we have

$$
\int_{N} \mathrm{~d} \chi_{u(t)} \circ v(t) \cdot U(t) \mathrm{d} t+\int_{N} \chi(u(t)) \cdot \operatorname{div} U(t) \mathrm{d} t=0 .
$$

It is not hard to see that, if $M$ is a Riemannian submanifold of a Euclidean space $E$, then $W^{1, q}(N, M)$ is just the set of the functions $u \in W^{1, q}(N, E)$ which satisfy $u(t) \in M$ for almost every $t$. We recall that smooth functions are not necessarily dense in these spaces if $q<n$.

\subsection{Young measures}

Let us denote by $J^{1}(N, M)$ the set of 1 -jets of maps $u: N \longrightarrow M$. In many examples, $N$ is an open subset of $\mathbb{R}^{n}, M$ is $\mathbb{R}^{m}$, and then

$$
J^{1}(N, M)=N \times \mathbb{R}^{m} \times \mathcal{L}\left(\mathbb{R}^{n}, \mathbb{R}^{m}\right) .
$$

We shall usually denote by $(t, x, v)$ the points of $J^{1}(N, M)$. We define the function

$$
r_{k}(t, x, v)=1+\|v\|+\left\|\wedge_{2} v\right\|+\cdots+\left\|\wedge_{k} v\right\|
$$

and associate to it the complete metric space $\mathcal{P}_{r_{k}}\left(J^{1}(N, M)\right)$ as in the appendix. This is the space of Borel probability measures $\eta$ on $J^{1}(N, M)$ such that $\int r_{k} d \eta<\infty$. We note that the measure on $N$ induced by the Riemann metric, which we have denoted by $\mathrm{d} t$, is finite, since $N$ is compact; to simplify the following definitions, we shall suppose that the measure of $N$ is 1 . 
Let $t: J^{1}(N, M) \longrightarrow N$ denote the natural projection; we denote by $\mathcal{Y}_{k}(N, M)$ the set of non-negative Borel measures $\eta \in \mathcal{P}_{r_{k}}\left(J^{1}(N, M)\right)$ such that $t_{\sharp} \eta$ coincides with the measure $\mathrm{d} t$. We endow $\mathcal{Y}_{k}(N, M)$ with the topology induced from $\mathcal{P}_{r_{k}}\left(J^{1}(N, M)\right)$.

Proposition 2. Assume that $L$ is a normal integrand which is bounded from below (or more generally such that $L / r_{k}$ is bounded from below). Then $\eta \longmapsto$ $\int L d \eta$ is lower semi-continuous on $\mathcal{Y}_{k}$.

Proof. Assume first that $L$ is continuous and that $L / r_{k}$ is bounded. Then, the functional is continuous by definition of the topology on $\mathcal{P}_{r_{k}}$.

As an intermediate step, assume that $L(t, x, v)$ is a Caratheodory integrand (measurable in $t$ and continuous in $(x, v)$ ) and that $L / r_{k}$ is bounded. By the Scorza-Dragoni Theorem, (see [5], Theorem I.1.1, p. 132) there exists an increasing sequence $K_{i}$ of compact subsets on $N$ such that $L$ is continuous on $J^{1}(N, M)_{\mid K_{i}}$ (the set of points $(t, x, v)$ such that $\left.t \in K_{i}\right)$ and such that $\cup_{i}\left(K_{i}\right)$ has full measure in $N$. Then, there exists a sequence of continuous functions $L_{i}$ such that $\left|L_{i}\right| / r_{k}$ is bounded, independently of $i$, and such that $L_{i}=L$ on $J^{1}(N, M)_{\mid K_{i}}$. It follows that the map $\eta \longmapsto \int L \mathrm{~d} \eta$ is the uniform limit on $\mathcal{Y}_{k}(N, M)$ of the continuous maps $\eta \longmapsto \int L_{i} \mathrm{~d} \eta$, and therefore it is continuous on $\mathcal{Y}_{k}(N, M)$.

In the general case, we first write the integrand $L(t, x, v)=r_{k}(t, x, v) g(t, x, v)$ with a normal integrand $g$ which is bounded from below. Then $g$ is the increasing pointwise limit of a sequence $g_{i}$ of bounded Caratheodory integrands, see [5], Theorem I.1.2, p. 138. Finally, the map $\eta \longmapsto \int L d \eta$ is the increasing limit of the continuous maps $\eta \longmapsto \int r_{k} g_{i} \mathrm{~d} \eta$, and therefore it is lower semicontinuous.

\subsection{Closed measures}

It is a fundamental and well known observation that there exists many nullLagrangians, that is functions $F(t, x, v): J^{1}(N, M) \longrightarrow \mathbb{R}$ such that

$$
\int_{N} F\left(t, u(t), \mathrm{d} u_{t}\right) \mathrm{d} t=0
$$

for all $C^{1}$ maps $u: N \longrightarrow M$. We define $\mathcal{N}_{k}(N, M)$ as the sets of continuous functions $F(t, x, v)$ such that

- $F / r_{k}$ is bounded,

- $\int_{N} F\left(t, u(t), \mathrm{d} u_{t}\right) \mathrm{d} t=0$ for each $C^{1} \operatorname{map} u$,

- There exists a compact $K \subset N$ such that $F(t, x, v)=0$ if $t \notin K$,

- We have $F(t, x, v)=\mathrm{F}_{t, x}\left(v, \wedge_{2} v, \ldots, \wedge_{k} v\right)=\mathrm{F}\left(t, x, v, \wedge_{2} v, \ldots, \wedge_{k} v\right)$, where $\mathrm{F}$ is continuous and where the functions $\mathrm{F}_{t, x}\left(v, v_{2}, \ldots, v_{k}\right)$ are affine (We say that $F_{t, x}$ is $k$-affine).

By extension, we shall also denote by $\mathcal{N}_{k}(N, M)$ the set of functions $\mathrm{F}\left(t, x, v_{1}, \ldots\right.$, $v_{k}$ ) associated with the elements $F \in \mathcal{N}_{k}(N, M)$. Note that the set $\mathcal{N}_{k}(N, M)$ may depend on the metric on $M$ if $M$ is not compact. 
Definition 3. A Young measure $\eta \in \mathcal{Y}_{k}$ is called closed if $\int F d \eta=0$ for all $F \in \mathcal{N}_{k}$. The set $\mathcal{C}_{k}$ of closed measures is closed in $\mathcal{Y}_{k}$, and contains the Young measures $\hat{u}$ associated with maps $u \in W^{1, n}(N, M)$.

Let us explain how to build null-Lagrangians. Given a field of $l$-vectors $U$, we will denote by $\dot{U}(t)$ the field of $(l-1)$-vectors which satisfies

$$
\mathrm{d}\left(i_{U} \Omega\right)=(-1)^{l+1} i_{\dot{U}} \Omega
$$

for any volume form $\Omega$ on $N$ which is compatible with the Riemannian metric (meaning that the volume of an orthonormal base is \pm 1 ). Notice that there are exactly two such volume forms on $N$ if it is orientable, and that they lead to the same $\dot{U}$. If $N$ is not orientable, then no global volume form $\Omega$ exists, but we can still define $\dot{U}$ by using volume forms defined on orientable open subsets of $N$ (for example discs). If $l=1$, for example, $U$ is a vector-field, and $\dot{U}=\operatorname{div} U$.

Lemma 4. For each $l \in 1, \ldots, k$, each smooth $(l-1)$-form $\chi$ on $M$ such that both $\chi$ and $\mathrm{d} \chi$ are bounded, and each compactly supported smooth field $U(t)$ of $l$-vectors on $N$, the function

$$
F(t, x, v):=\chi_{x} \circ \wedge_{l-1} v \cdot \dot{U}(t)+\mathrm{d} \chi_{x} \circ \wedge_{l} v \cdot U(t)
$$

belongs to $\mathcal{N}_{k}(N, M)$. In the case $l=1$, the form $\chi$ is just a function $\chi(x)$ on $M$, and the function $F$ can be rewritten more clearly

$$
F(t, x, v)=\chi(x) \operatorname{div} U(t)+\mathrm{d} \chi_{x} \circ v \cdot U(t) .
$$

Proof. Let $u: N \longrightarrow \mathbb{R}^{m}$ be a $C^{1}$ function. Let us still denote by $i_{U} \Omega$ and $\chi$ the pull-backs of $i_{U} \Omega$ and $\chi$ by the projections $N \times \mathbb{R}^{m} \longrightarrow N$ and $N \times \mathbb{R}^{m} \longrightarrow \mathbb{R}^{m}$ respectively. This allows us to define on $N \times \mathbb{R}^{m}$ the $(n-1)$-form $\xi=i_{U} \Omega \wedge \chi$. We have

$$
\begin{aligned}
0= & \int_{N}(I d \times u)^{*} \mathrm{~d} \xi=\int_{N} \mathrm{~d} \xi_{(t, u(t))} \circ \wedge_{n}\left(I d \times \mathrm{d} u_{t}\right) \cdot \lambda \mathrm{d} t \\
= & (-1)^{l+1} \int_{N}\left(i_{\dot{U}} \Omega \wedge \chi\right) \circ \wedge_{n}\left(I d \times \mathrm{d} u_{t}\right) \cdot \lambda \mathrm{d} t \\
& +(-1)^{n-l} \int_{N}\left(i_{U} \Omega \wedge \mathrm{d} \chi\right) \circ \wedge_{n}\left(I d \times \mathrm{d} u_{t}\right) \cdot \lambda \mathrm{d} t .
\end{aligned}
$$

Using Lemma 1 of Section 1.1, we obtain

$$
\begin{aligned}
0= & (-1)^{(l-1)(n-l)} \int_{N} \chi_{u(t)} \circ \wedge_{l-1} d u_{t} \cdot \dot{U}(t) \mathrm{d} t \\
& +(-1)^{(l+1)(n-l)} \int_{N} d \chi_{u(t)} \circ \wedge_{l} d u_{t} \cdot U(t) \mathrm{d} t .
\end{aligned}
$$

After simplifying the signs, we obtain

$$
\int_{N} \chi_{u(t)} \circ \wedge_{l-1} \mathrm{~d} u_{t} \cdot \dot{U}(t) \mathrm{d} t+\int_{N} d \chi_{u(t)} \circ \wedge_{l} \mathrm{~d} u_{t} \cdot U(t) \mathrm{d} t=0 .
$$

This is the required equality. 


\subsection{Generalized maps and Cartesian maps}

The closed measure $\eta \in \mathcal{C}_{k}$ is called a generalized map if there exists a measurable map $u: N \longmapsto M$ such that the marginal of $\eta$ on $N \times M$ is concentrated on the graph of $u$. We then say that $\eta$ is a generalized map over $u$. We denote by $\mathcal{G}_{k}(N, M)$ the set of generalized maps.

Definition 5. We denote by $\operatorname{cart}_{k}(N, M)$ the set of measurable maps $u$ such that there exists a generalized map over $u$. We call these maps cartesian maps. We have a natural projection $\pi$ from the set $\mathcal{G}_{k}(N, M)$ of generalized maps to the set $\operatorname{cart}_{k}(N, M)$ of cartesian maps.

The generalized maps have a remarkable structure:

Theorem 2. Let $\eta$ be a generalized map over $u$. Then, there exists a measurable family $\Gamma_{t}$ of probability measures on $\mathcal{L}\left(T_{t} N, T_{u(t)} M\right)$ such that $\eta=$ $d t \otimes \delta_{u(t)} \otimes \Gamma_{t}$. Setting

$$
g_{i}(t):=\int_{\mathcal{L}\left(T_{t} N, T_{u(t)} M\right)} \wedge_{j} v d \Gamma_{t}(v),
$$

we have $u \in W^{1,1}(N, M), g_{1}(t)=d u_{t}$ and $g_{i}(t)=\wedge_{i} g_{1}(t)$ for almost all $t$.

By Jensen's inequality, we immediately obtain:

Corollary 6. If $\eta$ is a generalized map over $u$, and if $L$ is $k$-convex, then

$$
\int_{J^{1}(N, M)} L d \eta \geqslant \int_{N} L\left(t, u(t), d u_{t}\right) d t
$$

Proof.

$$
\int_{J^{1}(N, M)} L \mathrm{~d} \eta=\int_{N} \int_{\mathcal{L}\left(T_{t} N, T_{u(t)} M\right)} L(t, u(t), v) \mathrm{d} \Gamma_{t}(v) \mathrm{d} t
$$

But we have, for each $t$,

$$
\begin{aligned}
\int_{\mathcal{L}\left(T_{t} N, T_{u(t)} M\right)} L(t, u(t), v) \mathrm{d} \Gamma_{t}(v) & =\int_{\mathcal{L}\left(T_{t} N, T_{u(t)} M\right)} \mathrm{L}\left(t, u(t), v, \wedge_{2} v, \ldots, \wedge_{k} v\right) \mathrm{d} \Gamma_{t}(v) \\
& \geqslant L\left(t, u(t), \mathrm{d} u(t), \wedge_{2} \mathrm{~d} u(t), \ldots, \wedge_{k} \mathrm{~d} u(t)\right)
\end{aligned}
$$

by Jensen's inequality, because $\int_{\mathcal{L}\left(T_{t} N, T_{u(t)} M\right)} \wedge_{j} v \mathrm{~d} \Gamma_{t}(v)=\wedge_{j} \mathrm{~d} u(t)$ by Theorem 2 .

The proof of Theorem 2 will occupy the end of the present section. The functions $g_{i}(t)$ depend only on the map $u$, not on $\eta$. This is a consequence of the following:

Lemma 7. Let $u: N \longrightarrow M$ be a given measurable function. Then there exists at most one family of functions $g_{1}(t), \ldots, g_{k}(t)$ such that

$$
\int_{N} F\left(t, u(t), g_{1}(t), g_{2}(t), \ldots, g_{k}(t)\right) d t=0
$$


for each $F \in \mathcal{N}_{k}$. We call these functions the distributional minors of $u$ if they exist. The map u belongs to $\operatorname{cart}_{k}(N, M)$ if and only if it admits distributional minors.

Proof. The maps $g_{l}$ satisfy the following equations:

$$
\int_{N} \mathrm{~d} \chi_{u(t)} \circ g_{1}(t) \cdot U(t) \mathrm{d} t+\int_{N} \chi(u(t)) \cdot \dot{U}(t) \mathrm{d} t=0
$$

for all smooth vector-field $U$ on $N$ supported in the interior of $N$, and all smooth function $\chi: M \longrightarrow \mathbb{R}$, and

$$
\int_{N} \mathrm{~d} \chi_{u(t)} \circ g_{l}(t) \cdot U(t) \mathrm{d} t+\int_{N} \chi(u(t)) \circ g_{l-1}(t) \cdot \dot{U}(t) \mathrm{d} t=0
$$

for all $l \in 2, \ldots, k$, all compactly supported smooth field of $l$-vectors $U(t)$ on $N$, and all smooth $l-1$-form $\chi$ on $M$ which is bounded as well as $\mathrm{d} \chi$. Now assume that $g_{l}^{\prime}(t)$ are other maps satisfying the same equation. Then, we have

$$
\int_{N} d \chi_{u(t)} \circ\left(g_{l}(t)-g_{l}^{\prime}\right) \cdot U(t)=0
$$

for each $l$, each $\chi$ and each $U$. We claim that this implies that $g_{l}(t)-g_{l}^{\prime}(t)=0$ almost everywhere. Since we have the freedom of choosing $U$, we conclude easily that $d \chi_{u(t)} \circ\left(g_{l}(t)-g_{l}^{\prime}(t)\right)=0$ for almost all $t$. If the claim did not hold, we could find a compact set $K \subset N$ of positive measure, such that $u$ and $g_{l}-g_{l}^{\prime}$ are continuous on $K$ and $g_{l}-g_{l}^{\prime}$ does not vanish on $K$. Let $t_{0}$ be a point of density of $K$, and let $\chi$ be a compactly supported $(l-1)$-form on $M$ such that

$$
d \chi_{u\left(t_{0}\right)} \circ\left(g_{l}\left(t_{0}\right)-g_{l}^{\prime}\left(t_{0}\right)\right) \neq 0 .
$$

Since $t_{0}$ is a density point of $K$, and since all the involved functions are continuous on $K$, there exists a compact subset $K^{\prime}$ of $K$ of positive measure such that the relation $d \chi_{u(t)} \circ\left(g_{l}(t)-g_{l}^{\prime}(t)\right) \neq 0$ holds for all $t \in K^{\prime}$. This is a contradiction.

Lemma 8. If $u \in \operatorname{cart}_{k}(N, M)$, then $u \in W^{1,1}(N, M)$ and the first distributional minor $g_{1}(t)$ of $u(t)$ is the weak derivative of $u$.

Proof. This is a direct consequence of (E1).

The following remark can be applied for example when $f$ is an embedding of $M$ into some Euclidean space, and $h$ is a chart of $N$ :

Proposition 9. Let $\tilde{N}$ and $\tilde{M}$ be other manifolds and let $f: M \longrightarrow \tilde{M}$ and $h: \tilde{N} \longrightarrow N$. Assume that $\tilde{M}$ is endowed with a complete metric. If $f$ is smooth with bounded differential, $h$ is a smooth diffeomorphism onto its image $h(\tilde{N}) \subset N$, and $u \in \operatorname{cart}_{k}(N, M)$, then $f \circ u \circ h \in \operatorname{cart}_{k}(\tilde{N}, \tilde{M})$. Moreover, the distributional minors $\tilde{g}_{i}$ of $f \circ u \circ h$ are:

$$
\tilde{g}_{i}(\tilde{t})=\wedge_{i} \mathrm{~d} f_{u(h(\tilde{t}))} \circ g_{i}(h(t)) \circ \wedge_{i} \mathrm{~d} h_{t},
$$

where $g_{i}$ are the distributional minors of $u$. 
Proof. Let us endow $\tilde{N}$ with the metric such that $h$ is an isometry. Let $\tilde{F}(t, x, v)$ be an element of $\mathcal{N}_{k}(\tilde{N}, \tilde{M})$. We want to prove that

$$
\int_{\tilde{N}} \tilde{F}\left(\tilde{t}, f \circ u \circ h(\tilde{t}), \mathrm{d} f_{u(h(\tilde{t}))} \circ \mathrm{d} u_{h(\tilde{t})} \circ d h_{\tilde{t}}\right) \mathrm{d} \tilde{t}=0 .
$$

Setting

$$
F(t, x, v):=\tilde{F}\left(h^{-1}(t), f(x), \mathrm{d} f_{x} \circ v \circ \mathrm{d} h_{t}\right)
$$

when $t \in h(\tilde{N})$ and $F(t, x, v)=0$ when $t \notin h(\tilde{N})$, we observe that (1) is equivalent to

$$
\int_{N} F\left(t, u(t), \mathrm{d} u_{t}\right) \mathrm{d} t=0
$$

This relation, on the other hand, holds if $F \in \mathcal{N}_{k}(N, M)$ by definition of $\operatorname{cart}_{k}(N, M)$.

We prove that $F \in \mathcal{N}_{k}(N, M)$. We begin to note that there is $\tilde{K} \subset \tilde{N}, \tilde{K}$ compact, such that $\tilde{F}(\tilde{t}, \tilde{x}, \tilde{v})=0$ if $\tilde{t} \notin \tilde{K}$; thus, $F(t, x, v)=0$ if $t$ does not belong to the compact set $h(\tilde{K})$. Moreover, (2) holds for all $C^{1}$ maps $u$. This is true because (2) is equivalent to (1), and (1) for $C^{1}$ maps follows because $\tilde{F}$ is a null Lagrangian, and thus it sends the $C^{1}$ map $f \circ u \circ h$ into zero; this amounts to (1) by the chain rule.

In order to prove the equality between distributional minors, we expand (1) to

$$
\int_{\tilde{N}} \tilde{\mathrm{F}}\left(\tilde{t}, f \circ u \circ h(\tilde{t}), \ldots, \wedge_{i} \mathrm{~d} f_{u(h(\tilde{t}))} \circ \tilde{g}_{i}(h(\tilde{t})) \circ \wedge_{i} \mathrm{~d} h_{\tilde{t}}, \ldots\right) \mathrm{d} \tilde{t}=0
$$

and use Lemma 7.

Lemma 10. We have $g_{l}(t)=\wedge_{l} g_{1}(t)$ for almost every $t \in N$.

Proof. If $M$ is a Riemannian submanifold of the Euclidean space $E$, then every map in $\operatorname{cart}_{k}(N, M)$ belongs to $\operatorname{cart}_{k}(N, E)$. Therefore, using the embedding theorem of Nash, we can assume for this proof that $M$ is a Euclidean space. The set of points $t_{0}$ which are simultaneously Lebesgue points of the function $u$ and of all the functions $g_{l}$, have total measure. Let $t_{0}$ be such a point. By taking a chart in $N$, we can suppose that $N$ is the ball $B$ of radius one in $\mathbb{R}^{n}$, that $t_{0}=0$, and that $\mathrm{d} t$ is the Lebesgue measure. Translating in $\mathbb{R}^{n}$, we can suppose that $u(0)=0$. Let us consider, for $s \geqslant 1$ the maps

$$
u^{s}(t):=s u(t / s), \quad g_{l}^{s}:=g_{l}(t / s)
$$

on $N$. By Proposition $9, u^{s}$ is a cartesian map on $\tilde{N}$, the ball of radius $s$, and $g_{l}^{s}$ are its distributional minors. Our hypothesis on the point $t_{0}$ can be rephrased by saying that, strongly in $L^{1}(N)$, we have

$$
u^{s}(t) \longrightarrow u^{\infty}(t)=g_{1}(0) t, \quad g_{l}^{s}(t) \longrightarrow g_{l}^{\infty}(t)=g_{l}(0)
$$

when $s \longrightarrow \infty$. We can take a subsequence in order that these limits also hold almost everywhere. Let $F$ be a null Lagrangian on the ball of radius 1 ; in 
particular, when trivially extended, it is a null Lagrangian on the ball of radius $s$, so that

$$
\int_{N} F\left(t, u^{s}(t), \mathrm{d} u^{s}(t)\right) \mathrm{d} t=0
$$

Passing to the limit, we obtain

$$
\int_{N} \mathrm{~F}\left(t, u^{\infty}(t), g_{1}^{\infty}(t), \ldots, g_{k}^{\infty}(t)\right) \mathrm{d} t=0 .
$$

In other words, the limit function $u^{\infty}$ has $g_{l}^{\infty}$ as distributional minors. On the other hand, since the function $u^{\infty}$ is smooth, we know that its distributional minors are $\wedge_{l} d u^{\infty}(t)$, which here are just the constant functions $\wedge_{l} g_{1}(0)$. Therefore, by uniqueness of the distributional minors, we have proved that $\wedge_{l} g_{1}(0)=g_{l}(0)$.

We have proved Theorem 2. We can reformulate it as follows: A function $u$ belongs to $\operatorname{cart}_{k}(N, M)$ if and only if the minors $\wedge_{l} d u$ belong to $L^{1}$ and are distributional, which means that they satisfy the equation

$$
\int_{N} \mathrm{~F}\left(t, u(t), d u_{t}, \wedge_{2} d u_{t}, \ldots, \wedge_{k} d u_{t}\right) \mathrm{d} t=0
$$

for all $\mathrm{F} \in \mathcal{N}_{k}$. Note that $\operatorname{cart}_{1}(N, M)=W^{1,1}(N, M)$.

\subsection{Topology}

The set $\mathcal{G}_{k}$ of generalized maps is endowed with the topology of $\mathcal{Y}_{k}$.

Proposition 11. The set $\mathcal{G}_{k}(N, M)$ of generalized maps is closed in $\mathcal{Y}_{k}(N, M)$.

Proof. Let $\eta^{j}$ be a sequence of generalized maps above $u^{j}$. Let us assume that the sequence $\eta^{j}$ is converging to $\eta$ in $\mathcal{C}_{k}(N, M)$. We have to prove that there exists a map $u \in W^{1,1}(N, M)$ such that the marginal of $\eta$ on $N \times M$ is concentrated on the graph of $u$. It is enough to prove that, for each embedded ball $B \subset N$, the marginal of $\eta_{\mid J^{1}(B, M)}$ on $B \times M$ is concentrated on the graph of a map $u$. As a consequence, we can suppose that $N$ is the open unit ball in $\mathbb{R}^{n}$. We consider $M$ as a Riemannian submanifold of a Euclidean space $E$, so that we see $u^{j}$ as elements of $W^{1,1}(N, E)$ with values in $M$. Let $m^{j} \in E$ be the average of $u^{j}, m^{j}=\int_{N} u_{j}(t) \mathrm{d} t$. Since the sequence $\eta^{j}$ is $r_{k}$-tight, see Appendix, the derivatives $\mathrm{d} u^{j}$ are bounded in $L^{1}$. Therefore, by the Poincaré inequality, the sequence $\left(u^{j}-m^{j}\right)$ is bounded in $W^{1,1}$. By the compactness of the embedding $W^{1,1} \longrightarrow L^{1}$, this sequence is strongly compact in $L^{1}$. We assume, taking a subsequence, that it has a limit $u^{\infty}$, and that the convergence holds almost everywhere. By Lusin and Egorov Theorems, for all $\epsilon>0$, there exists a compact subset $K \subset N$ such that $\mathrm{d} t(N-K) \leqslant \epsilon$ and such that $u^{j}$ is continuous on $K$ and $\left(u^{j}-m^{j}\right)$ is converging uniformly on $K$ to $u^{\infty}$. It is clear at this point that the unboundedness of $m^{j}$ would contradict the tightness of $\eta^{j}$, and therefore we can assume that the averages $m^{j}$ have a limit $m^{\infty}$. Setting $u:=u^{\infty}+m^{\infty}$, we see that $u^{j}$ is converging uniformly to the 
continuous function $u$ on $K$. Denoting by $\mu$ the marginal of $\eta$ on $N \times M$, we conclude that the $\mu$-measure of the graph of $u$ is greater that $1-\epsilon$. Since this holds for all $\epsilon>0$, we conclude that the measure $\mu$ is concentrated on the graph of $u$.

\section{Boundary conditions and compactness}

In most applications, the manifold $M$ is not compact, and it is necessary to introduce boundary conditions in order to get compactness. We fix, as explained in the introduction, a map $u_{0} \in \operatorname{cart}_{k}(N, M)$. We define the set $\mathcal{C}_{k}\left(N, M ; u_{0}\right) \subset \mathcal{C}_{k}(N, M)$ of closed measures with boundary $u_{0}$ as the set of measures $\eta \in \mathcal{C}_{k}(N, M)$ such that

$$
\begin{aligned}
& \int_{J^{1}(N, M)} \mathrm{d} \chi_{x} \circ v \cdot U(t)+\chi(x) \cdot \operatorname{div} U(t) \mathrm{d} \eta(t, x, v) \\
= & \int_{N} \mathrm{~d} \chi_{u_{0}(t)} \circ \mathrm{d} u_{0}(t) \cdot U(t)+\chi\left(u_{0}(t)\right) \cdot \operatorname{div} U(t) \mathrm{d} t
\end{aligned}
$$

for each smooth vectorfield $U(t)$ on $\bar{N}$ (not necessarily supported in a compact set of $N$ ) and each bounded smooth function $\chi(x)$ on $M$ with bounded derivative. We can also define the set of generalized maps with boundary value $u_{0}$ :

$$
\mathcal{G}_{k}\left(N, M ; u_{0}\right):=\mathcal{G}_{k}(N, M) \cap \mathcal{C}_{k}\left(N, M ; u_{0}\right) .
$$

The space $\operatorname{cart}_{k}\left(N, M ; u_{0}\right)$ is the space of maps $u$ such that there exists a generalized map $\eta \in \mathcal{G}_{k}\left(N, M ; u_{0}\right)$ above $u$, or in other words the maps $u$ whose Young measure $\hat{u}$ belongs to $\mathcal{G}_{k}\left(N, M ; u_{0}\right)$. In [12], the functions in $\operatorname{cart}_{k}\left(N, M ; u_{0}\right)$ are said to satisfy a weak anchorage condition. In the case where $M$ is a Euclidean space, the functions in $\operatorname{cart}_{k}\left(N, M ; u_{0}\right)$ are just the functions in $\operatorname{cart}_{k}(N, M)$ which have the same trace on $\partial N$ as $u_{0}$ in the $W^{1,1}$ sense.

Proposition 12. Let $L$ be a $k$-convex and coercive Lagrangian. For each $c>0$, the set of measures $\eta \in \mathcal{C}_{k}\left(N, M ; u_{0}\right)$ which satisfy

$$
\int_{J^{1}(N, M)} L(t, x, v) \mathrm{d} \eta(t, x, v) \leqslant c
$$

is compact.

Proof. Let us denote by $\mathcal{C}(c)$ the set of measures $\eta \in \mathcal{C}_{k}\left(N, M ; u_{0}\right)$ which satisfy (3). Since the functional

$$
\eta \longmapsto \int L d \eta
$$

is lower semi-continuous on $\mathcal{Y}_{k}(N, M)$ (by Proposition 2$)$, and since $\mathcal{C}_{k}\left(N, M ; u_{0}\right)$, is closed in $\mathcal{Y}_{k}(N, M)$, the set $\mathcal{C}(c)$ is closed in $\mathcal{Y}_{k}(N, M)$. So it is enough to prove that it is relatively compact. By the appendix, this follows if we can 
prove that it is $r_{k}$-tight. In other words, we have to show that for each $\epsilon>0$ there exists a compact subset $Z \in J^{1}(N, M)$ such that

$$
\int_{J^{1}(N, M) \backslash Z(R)} r_{k}(t, x, v) \mathrm{d} \eta(t, x, v) \leqslant 2 \epsilon
$$

for each measure $\eta \in \mathcal{C}(c)$. We shall prove that this holds for

$$
Z(R)=\left\{(t, x, v) \in J^{1}(N, M) \quad: \quad d\left(x_{0}, x\right) \leqslant R,\|v\| \leqslant R\right\}
$$

when $R$ is large enough $\left(x_{0}\right.$ is a point in $M$ that we have fixed once and for all). At this point it is convenient to assume, without loss of generality, that $L \geqslant 0$. We define

$$
\tilde{Z}(R)=\left\{(t, x, v) \in J^{1}(N, M) \quad: \quad\|v\| \leqslant R\right\}
$$

and we see that there exists $A(R)>0$ with $A(R) \rightarrow+\infty$ as $R \rightarrow+\infty$ such that, for all $\eta \in \mathcal{C}(c)$,

$c \geq \int_{J^{1}(N, M) \backslash \tilde{Z}(R)} L(t, x, v) \mathrm{d} \eta(t, x, v) \geqslant A(R) \int_{J^{1}(N, M) \backslash \tilde{Z}(R)} r_{k}(t, x, v) \mathrm{d} \eta(t, x, v)$.

Taking $R$ sufficiently large, we get from the inequality above that

$$
\int_{J^{1}(N, M) \backslash \tilde{Z}(R)} r_{k}(t, x, v) \mathrm{d} \eta(t, x, v) \leqslant \epsilon
$$

for each $\eta \in \mathcal{C}(c)$. Setting now

$$
\hat{Z}(R)=\left\{(t, x, v) \in J^{1}(N, M) \quad: \quad d\left(x_{0}, x\right) \leqslant R\right\}
$$

we see that the desired inequality follows if we prove that

$$
\eta\left(J^{1}(N, M)-\hat{Z}(R)\right) \leqslant \epsilon(R) \quad \forall \eta \in \mathcal{C}(c)
$$

for all $R$, with $\epsilon(R) \longrightarrow 0$ as $R \longrightarrow \infty$. Indeed, taking $R_{0}$ such that (4) holds, and then setting $S=\max _{\tilde{Z}\left(R_{0}\right)} r_{k}$, we get

$\int_{J^{1}(N, M) \backslash Z(R)} r_{k} \mathrm{~d} \eta \leqslant \int_{J^{1}(N, M) \backslash \tilde{Z}\left(R_{0}\right)} r_{k} \mathrm{~d} \eta+S \eta\left(J^{1}(N, M) \backslash \hat{Z}(R)\right) \leqslant \epsilon+S \epsilon(R)$.

In order to prove (5), we consider, for each $R>0$, a function $g \in C^{1}(M, \mathbb{R})$ such that

$$
\begin{gathered}
0 \leqslant g_{R}(x) \leqslant 1, \quad g(x)=1 \quad \text { if } \quad d\left(x_{0}, x\right) \geqslant R, \\
g(x)=0 \quad \text { if } \quad d\left(x_{0}, x\right) \leqslant R / 2 \\
\left|d g_{x}\right| \leqslant \delta(R) \quad \forall x,
\end{gathered}
$$

where $\delta(R) \longrightarrow 0$ as $R \longrightarrow \infty$; and a smooth vector-field $U(t)$ on $\bar{N}$ such that $\dot{U}=1$ on $N$ or equivalently such that $\operatorname{div} U=1$ on $N$. The existence of such 
a vector-field is given by Lemma 13 below. We note that $U$ is bounded, since $\bar{N}$ is compact. Since $\mathcal{C}(c) \subset \mathcal{C}_{k}\left(N, M ; u_{0}\right)$, we have, for $\eta \in \mathcal{C}(c)$,

$$
\begin{aligned}
\int_{J^{1}(N, M)} g(x) \mathrm{d} \eta(t, x, v)= & \int_{N} g\left(u_{0}(t)\right)+\mathrm{d} g_{u_{0}(t)} \circ \mathrm{d} u_{0}(t) \cdot U(t) \mathrm{d} t \\
& -\int_{J^{1}(N, M)} \mathrm{d} g_{x} \circ v \cdot U(t) \mathrm{d} \eta(t, x, v) .
\end{aligned}
$$

The last formula and the definition of $g$ imply that there exists $C>0$ such that

$$
\int_{J^{1}(N, M) \backslash Z(R)} \mathrm{d} \eta(t, x, v) \leqslant C \delta(R)+\int_{N} g\left(u_{0}(t)\right) \mathrm{d} t
$$

for all $R$ and all $\eta \in \mathcal{C}(c)$. The term on the right converges to zero as $R \longrightarrow \infty$, this ends the proof.

Lemma 13. Let $\bar{N}$ be a compact Riemannian manifold with a non-empty boundary. There exists a smooth vector-field $U(t)$ on $\bar{N}$ such that $\dot{U}=1$ on $N$ or equivalently such that $\operatorname{div} U=1$ on $N$.

Proof. In the case where $N$ is a ball in $\mathbb{R}^{n}$, this is obvious, just take $U(t)=t / n$. In general, one can build $U$ as the gradient of a function $h$ which solves $\Delta h=1$ on $N$.

\section{Conclusion}

We now collect the tools we have introduced to prove Theorem 1. We also add some discussions and variations.

\subsection{Proof of Theorem 1}

By Propositions 11 and 12, there exists a generalized map $\eta$ over some $u \in W^{1}(N, M)$ such that $\eta$ minimizes $\int L \mathrm{~d} \eta$ on $\mathcal{G}_{k}\left(N, M ; u_{0}\right)$. We want to show that $u$ minimizes in $\operatorname{cart}_{k}\left(N, M ; u_{0}\right)$.

If $v \in \operatorname{cart}_{k}\left(N, M ; u_{0}\right)$ is another map, we have

$$
\int_{J^{1}(N, M)} L\left(t, u(t), \mathrm{d} u_{t}\right) \mathrm{d} t \leqslant \int L \mathrm{~d} \eta \leqslant \int L \mathrm{~d} \hat{v}=\int_{J^{1}(N, M)} L\left(t, v(t), \mathrm{d} v_{t}\right) \mathrm{d} t,
$$

where the first inequality comes from Corollary 6 , and where $\hat{v} \in \mathcal{G}_{k}\left(N, M ; u_{0}\right)$ is the Young measure associated with $v$. This proves that $u$ is minimizing in $\operatorname{cart}_{k}\left(N, M ; u_{0}\right)$.

\subsection{An example}

We consider $M=\mathbb{R}^{2} ; N=B$, the unit open ball of $\mathbb{R}^{2}$, and the Lagrangian

$$
L(t, x, v)=\epsilon\left(|v|^{p}+|t|^{4}|v|^{4}\right)+|\operatorname{det} v|^{2},
$$

with $p \in] 1,2[$. We claim that

$$
\inf _{u \in W^{1,1}(N, M ; I d)} \int_{N} L\left(t, u(t), \mathrm{d} u_{t}\right) \mathrm{d} t<\min _{u \in \operatorname{cart}_{2}(N, M ; I d)} \int_{N} L\left(t, u(t), \mathrm{d} u_{t}\right) d t
$$


when $\epsilon>0$ is small enough. Indeed, taking $\underline{u}(t)=t /|t|$, and observing that $\operatorname{det} \mathrm{d} \underline{u}=0$, we get a constant $C>0$ such that

$$
\inf _{u \in W^{1,1}(N, M ; I d)} \int_{N} L\left(t, u(t), \mathrm{d} u_{t}\right) \mathrm{d} t \leqslant \int_{N} L\left(t, \underline{u}(t), \mathrm{d} \underline{u}_{t}\right) \mathrm{d} t \leqslant C \epsilon .
$$

On the other hand, if $u \in \operatorname{cart}_{2}\left(B, \mathbb{R}^{2} ; I d\right)$ is a minimizer we have

$$
\int_{B} L\left(t, u(t), \mathrm{d} u_{t}\right) \mathrm{d} t \geqslant \int_{B}\left|\operatorname{det} \mathrm{d} u_{t}\right|^{2} \mathrm{~d} t \geqslant \frac{1}{|B|}\left(\int_{B}\left|\operatorname{det} \mathrm{d} u_{t}\right| \mathrm{d} t\right)^{2} \geqslant \pi,
$$

where the last inequality follows from the following lemma:

Lemma 14. If $u \in \operatorname{cart}_{2}\left(B, \mathbb{R}^{2} ;\right.$ Id) minimizes $\int_{B} L\left(t, u(t), \mathrm{d} u_{t}\right) \mathrm{d} t<\infty$, then $\int_{B} \operatorname{det} \mathrm{d} u(t) \mathrm{d} t=\pi$.

Proof. We claim that $u(B) \subset \bar{B}$. Indeed, let $f: \mathbb{R}^{2} \longrightarrow \mathbb{R}^{2}$ be a smooth diffeomorphism such that $f=I d$ on $B$ and $|\mathrm{d} f|<1$ outside of $\bar{B}$. By Proposition 9, the map $f \circ u$ belongs to $\operatorname{cart}_{2}\left(B, \mathbb{R}^{2}\right)$, and it has the same boundary condition as $u$. Since $|\mathrm{d} f(u)| \leqslant 1$ we have that $|\mathrm{d}(f \circ u)| \leqslant|\mathrm{d} u|$ and $|\operatorname{det} \mathrm{d}(f \circ u)| \leqslant|\operatorname{det} \mathrm{d} u|$; the first inequality is strict if $|u|>1$ and $\mathrm{d} u \neq 0$. If we did not have $u(t) \subset \bar{B}$ for almost every $t$, the action of $f \circ u$ would be strictly smaller than the action of $u$, which would contradict the assumption that $u$ is a minimizer.

Let us denote by $A$ the annulus $1 / 2<|t|<1$. We have

$$
u \in W^{1, p}\left(B, \mathbb{R}^{2}\right) \cap W^{1,4}\left(A, \mathbb{R}^{2}\right),
$$

so that $u$ is continuous on $A$, and extends by continuity to $\partial B$, where it takes the value $\left.u\right|_{\partial B}=I d$. Finally, recall that $u(B) \subset \bar{B}$. Define

$$
u_{i}(t):=i^{2} \int_{B} \tau(i s) u((1-1 / i) t-s) \mathrm{d} s,
$$

where $\tau: B \longrightarrow[0,1]$ is a smooth convolution kernel. It is classical that $u_{i} \longrightarrow u$ in $W^{1, p}\left(B, \mathbb{R}^{2}\right)$, and in $W^{1,4}(A, \mathbb{R})$. As a consequence, $\left.u_{i}\right|_{\partial B}$ converges uniformly to the identity; since $u_{i}$ is smooth, this implies

$$
\int_{B} \operatorname{det} \mathrm{d} u_{i} \mathrm{~d} t \longrightarrow \pi
$$

Thus it is enough to prove that

$$
\int_{B} \operatorname{det} \mathrm{d} u_{i} \mathrm{~d} t \longrightarrow \int_{B} \operatorname{det} \mathrm{d} u \mathrm{~d} t
$$

Let $r \in] 1 / 2,1\left[\right.$ and let $\phi_{r} \in C_{0}^{\infty}(B, \mathbb{R})$ be such that $0 \leqslant \phi_{r} \leqslant 1$ and $\phi_{r}=1$ on $B(0, r)$. Since

$$
\int_{B} \operatorname{det} \mathrm{d} u \cdot \phi_{r} \mathrm{~d} t \longrightarrow \int_{B} \operatorname{det} \mathrm{d} u \mathrm{~d} t
$$

as $r \longrightarrow 1$, the formula (6) follows if we prove

$$
\left.\int_{B} \operatorname{det} \mathrm{d} u_{i} \cdot \phi_{r} \mathrm{~d} t \longrightarrow \int_{B} \operatorname{det} \mathrm{d} u \cdot \phi_{r} \mathrm{~d} t \quad \forall r \in\right] 1 / 2,1[
$$


and

$$
\left|\int_{B} \operatorname{det} \mathrm{d} u_{i} \cdot\left(1-\phi_{r}\right) \mathrm{d} t\right|<\epsilon \quad \forall i \quad \text { if } \quad r \geq 1-\delta .
$$

Note that (8) follows from the boundedness of $\operatorname{det} \mathrm{d} u_{i}$ in $L^{2}(A)$. In order to prove $(7)$, we set $u_{i}=\left(u_{i}^{1}, u_{i}^{2}\right)$ and $u=\left(u^{1}, u^{2}\right)$, we call $\left(x_{1}, x_{2}\right)$ the coordinates on the target space $\mathbb{R}^{2}$ and we assert that

$$
\int_{B} \operatorname{det} \mathrm{d} u \cdot \phi_{r} \mathrm{~d} t=\int_{B} u^{1}\left(\partial_{2} u^{2},-\partial_{1} u^{2}\right) \cdot \nabla \phi_{r} \mathrm{~d} t .
$$

Indeed, this formula is just (El) with $l=2, \chi=a\left(x^{1}\right) \mathrm{d} x^{2}$ and $U=\phi_{r} e_{1} \wedge e_{2}$, where $a: \mathbb{R} \longrightarrow[-3,3]$ is a smooth function such that $a\left(x^{1}\right)=x^{1}$ on $[-2,2]$. Here we use that $u(B) \subset \bar{B}$. Similarly, by Lemma 4 ,

$$
\int_{B} \operatorname{det} \mathrm{d} u_{i} \cdot \phi_{r} \mathrm{~d} t=\int_{B} u_{i}^{1}\left(\partial_{2} u_{i}^{2},-\partial_{1} u_{i}^{2}\right) \cdot \nabla \phi_{r} \mathrm{~d} t
$$

As a consequence $(7)$ is equivalent to

$$
\int_{B}\left[u_{i}^{1}\left(\partial_{2} u_{i}^{2},-\partial_{1} u_{i}^{2}\right)\right] \cdot \nabla \phi_{r} \mathrm{~d} t \longrightarrow \int_{B}\left[u^{1}\left(\partial_{2} u^{2},-\partial_{1} u^{2}\right)\right] \cdot \nabla \phi_{r} \mathrm{~d} t
$$

which holds because the integrand is converging almost everywhere and is bounded in $L^{2}$.

\subsection{Weak continuity of minors}

Let us mention the following classical result which follows from our tools (see [12], 3.3.1 or [10], 8.3):

Proposition 15. Let $N$ be a bounded disc in $\mathbb{R}^{n}$. Let $u_{i}$ be a sequence of maps in $\operatorname{cart}_{k}\left(N, \mathbb{R}^{m}\right)$, and let $u \in W^{1,1}(N, M)$ and $g_{j}(t) \in L^{1}\left(N, \mathcal{L}\left(\wedge_{j} \mathbb{R}^{n}, \wedge_{j} \mathbb{R}^{m}\right)\right)$ be such that $u_{i} \longrightarrow u$ weakly in $W^{1,1}$ and

$$
\wedge_{2} \mathrm{~d} u_{i} \longrightarrow g_{2}, \ldots, \wedge_{k} \mathrm{~d} u_{i} \longrightarrow g_{k}
$$

weakly in $L^{1}$. Then $g_{2}=\wedge_{2} \mathrm{~d} u, \ldots, g_{k}=\wedge_{k} \mathrm{~d} u$.

Proof. We consider the Young measures $\hat{u}_{i}$ in $\mathcal{G}_{k}(N, M)$ associated with the functions $u_{i}$. Now weak convergence implies uniform integrability, which translates to the fact that $\hat{u}_{i}$ is $r_{k}$-tight, and therefore compact in $\mathcal{Y}_{k}\left(N, \mathbb{R}^{m}\right)$. We can suppose that it has a limit $\eta$, which is a generalized map above $u$. If $F(t, v)=\mathrm{F}\left(t, v, v_{2}, \ldots, v_{k}\right)$ is a continuous function which is affine in $\left(v, v_{2}, \ldots, v_{k}\right)$, then we have

$$
\int F \mathrm{~d} \hat{u}_{i} \longrightarrow \int F \mathrm{~d} \eta
$$

because $\hat{u}_{i} \longrightarrow \eta$. On the other hand, since $F$ is affine in the minors, and since $\wedge_{j} \mathrm{~d} u_{i} \longrightarrow g_{j}$ weakly, we have

$$
\int \mathrm{F}\left(t, \mathrm{~d} u_{i}(t), \wedge_{2} \mathrm{~d} u_{i}(t), \ldots, \wedge_{k} \mathrm{~d} u_{i}(t)\right) \mathrm{d} t \longrightarrow \int \mathrm{F}\left(t, \mathrm{~d} u(t), g_{2}(t), \ldots g_{k}(t)\right) d t .
$$


We conclude that

$$
\int F \mathrm{~d} \eta=\int \mathrm{F}\left(t, \mathrm{~d} u(t), g_{2}(t), \ldots g_{k}(t)\right) d t
$$

This implies that, for almost all $t$,

$$
g_{j}(t)=\int \wedge_{j} v \mathrm{~d} \Gamma_{t}=\wedge_{j} \mathrm{~d} u(t)
$$

by Theorem 2 .

\subsection{On null-Lagrangians}

It may seem unnatural in the definitions of the sets $\mathcal{N}_{k}(N, M)$ to require that the null-Lagrangians $F(t, x, v)$ be $k$-affine functions of $v$. Indeed, working with a larger set $\mathcal{N}(N, M)$ of null-Lagrangians would make the result stronger, and may allow to relax somewhat the $k$-convexity hypothesis on $L$. The following result, however, shows that there is not much hope in that direction:

Proposition 16. Let us assume that $N=\mathbb{R}^{n}$, and that $M=\mathbb{R}^{m}$. If $F$ is a null-Lagrangian such that $F / r_{k}$ is bounded, then $F_{t, x}$ is $k$-affine for each $t$ and $x$.

Proof. We just give an idea of the proof. It follows from Proposition 9 that, for $\lambda>0$ and $\left(t_{0}, x_{0}\right) \in \mathbb{R}^{n} \times \mathbb{R}^{m}$, the function

$$
F_{\lambda}(t, x, v):=F\left(t_{0}+\lambda t, x_{0}+\lambda x, v\right)
$$

is a null-Lagrangian. But then $F_{0}$ is also a null-Lagrangian, which means that $F_{t_{0}, x_{0}}$ is quasi-affine in the sense of [10], Section 4.1 ; but in the same section of [10] it is proven that quasi-affine functions are poly-affine. In other words, there exists an affine function $\mathrm{F}\left(v, v_{2}, \ldots, v_{p}\right)$ such that $F_{t_{0}, x_{0}}(v)=$ $\mathrm{F}\left(v, \wedge_{2} v, \ldots, \wedge_{p} v\right)$, where $p=\min \{m, n\}$. But the bound implies that $\mathrm{F}$ does not depend on $\wedge_{j} v$ for $j>k$.

\subsection{More general setting}

The heart of the matters is the Jensen's inequality obtained in Corollary 6 . This inequality is the result of an equilibrium between the known properties of the measures $\Gamma_{t}$ appearing in the disintegration of generalized maps and the convexity assumed on the integrand $L$.

Other, but less explicit equilibria might be obtained as follows. Let $r(t, x, v)$ be a continuous function on $J^{1}(N, M)$ such that $r(t, x, v) \geqslant 1+\|v\|$. We define the associated Kantorovich-Rubinstein space $\mathcal{P}_{r}\left(J^{1}(N, M)\right)$, which is the set of Borel probabilities $\eta$ on $J^{1}(N, M)$ such that $\int r \mathrm{~d} \eta<\infty$. We also define the set $\mathcal{Y}_{r}(N, M)$ of those elements $\eta$ of $\left.\mathcal{P}_{r}\left(J^{1}(N, M)\right)\right)$ such that $t_{\sharp} \eta=\mathrm{d} t$.

Now let $\hat{\mathcal{G}}_{r}(N, M)$ be the closure, in $\mathcal{Y}_{r}(N, M)$ of the set of Young measures associated with smooth maps. We can prove as in Proposition 11 that, to each $\eta \in \hat{\mathcal{G}}_{r}(N, M)$ is associated a map $u \in W^{1,1}(N, M)$ such that

$$
\eta=\mathrm{d} t \otimes \delta_{u(t)} \otimes \Gamma_{t}
$$


and such that $\int v \mathrm{~d} \Gamma_{t}=\mathrm{d} u(t)$ for almost all $t$. We can define $\operatorname{cart}_{r}(N, M)$ as the set of maps which appear in this way. In this setting, we can fix boundary conditions as before by taking $u_{0} \in \operatorname{cart}_{r}(N, M)$. If the coercivity condition of the Lagrangian is modified to

$$
L(t, x, v) \geqslant l(r(t, x, v)),
$$

with $l$ super-linear, we still have compactness: Proposition 12 still holds, with the same proof. So if $L$ is a normal integrand satisfying the modified coercivity condition, then there exists a Young measure $\eta \in \hat{\mathcal{G}}_{r}\left(N, M ; u_{0}\right)$ which minimizes the integral $\int L d \eta$ in this set.

In order to prove the existence of minimizers in $\operatorname{cart}_{r}\left(\tilde{N}, M ; u_{0}\right)$, it is enough to adapt the convexity condition, in such a way that Corollary 6 holds for the elements of $\hat{\mathcal{G}}_{r}(N, M)$.

Let $\mathcal{P}_{t, x}$ be the set of Borel probability measures $\Gamma$ on $\mathcal{L}\left(T_{t} N, T_{x} M\right)$ such that $\int r_{t, x}(v) d \Gamma(v)<\infty$. In short, we have

$$
\mathcal{P}_{t, x}:=\mathcal{P}_{r_{t, x}}\left(\mathcal{L}\left(T_{t} N, T_{x} M\right)\right)
$$

(see the Appendix below). Let $B$ be a closed ball of volume one in $T_{t} N$. Let $\mathrm{P}_{t, x}$ be the closure, in $\mathcal{P}_{t, x}$ of the measures of the form

$$
\Gamma=(\mathrm{d} u)_{\sharp}\left(\mathrm{d} t_{\mid B}\right),
$$

where $u: T_{t} N \longrightarrow T_{x} M$ is a smooth map supported in $B$. Note that if $\Gamma \in \mathrm{P}_{t, x}$, then $\int_{\mathcal{L}\left(T_{t} N, T_{x} M\right)} v \mathrm{~d} \Gamma=0$. A last notation is necessary: we denote by $\tau_{z}$ the translation of vector $z$. Then, possibly under some mild assumption on the function $r$, the following result can be proved by a blow-up argument called localisation procedure in [14]:

Structure Theorem. The measures $\eta \in \hat{\mathcal{G}}_{r}(N, M)$ can be written in the form (9), with

$$
\left(\tau_{-\mathrm{d} u_{t}}\right)_{\sharp} \Gamma_{t} \in \mathrm{P}_{t, x}
$$

for almost all $t$.

As a consequence, the convexity condition that has to be assumed in order that Corollary 6, and then Theorem 1 hold in this more general setting is

$$
\int_{\mathcal{L}\left(T_{t} N, T_{x} M\right)} L_{t, x}(a+v) \mathrm{d} \Gamma(v) \geqslant L(t, x, a)
$$

for all $(t, x) \in N \times M$, for all $a \in \mathcal{L}\left(T_{t} N, T_{x} M\right)$ and for all $\Gamma \in \mathrm{P}_{t, x}$. This is not an easy condition to check on examples.

\section{Appendix. Kantorovich-Rubinstein space}

Let us recall some standard facts on probability measures, see $[2,16]$. Let $(X, d)$ be a complete and separable metric space, and let $r: X \longrightarrow[1, \infty)$ be a 
continuous function. Let $\mathcal{P}_{r}(X)$ be the set of Borel probability measures $\mu$ on $X$ which satisfy

$$
\int_{X} r(x) d \mu(x)<\infty .
$$

Let us denote by $C_{r}(X)$ the set of continuous functions $f$ on $X$ such that

$$
\sup _{x \in X} \frac{|f(x)|}{r(x)}<\infty .
$$

There exists a distance $d$ on $\mathcal{P}_{r}(X)$ such that $d\left(\mu_{n}, \mu\right) \longrightarrow 0$ if and only if

$$
\int f d \mu_{n} \longrightarrow \int f d \mu
$$

for all $f \in C_{r}(X)$. This distance can be chosen such that, in addition, the metric space $\left(\mathcal{P}_{r}, d\right)$ is a complete and separable metric space.

In order to define such a distance $d$ on $\mathcal{P}_{r}(X)$ one can define first the distance

$$
d_{r}(x, y):=\min (d(x, y), 1)+|r(y)-r(x)|
$$

on $X$, which is complete and equivalent to $d$. Then, we can define the distance $d$ on $\mathcal{P}_{r}(X)$ as the Kantorovich-Rubinstein (also called 1-Wasserstein) distance of $\left(X, d_{r}\right)$.

The relatively compact subsets of $\left(\mathcal{P}_{r}(X), d\right)$ are those which are $r$-tight:

Definition 17. The subset $Y \subset \mathcal{P}_{r}(X)$ is called $r$-tight if one of the following equivalent properties holds:

- For each $\epsilon>0$, there exists a compact set $K \subset X$ such that

$$
\int_{X-K} r(x) d \mu \leqslant \epsilon
$$

for each $\mu \in Y$.

- There exists a function $f: X \longrightarrow[0, \infty]$ whose sublevels are compact and a constant $C$ such that $\int_{X} r(x) f(x) d \mu \leqslant C$ for each $\mu \in Y$.

- The family $Y$ is tight and $r$ is $Y$-uniformly integrable. The first means that, for each $\epsilon>0$, there exists a compact set $K \subset X$ such that $\mu(X-K) \leqslant \epsilon$ for each $\mu \in Y$. The second means that for each $\epsilon>0$, there exists a ball $B$ in $X$ such that $\int_{X-B} r(x) d \mu \leqslant \epsilon$ for each $\mu \in Y$.

Note that 1-tightness is just tightness if $r \equiv 1$. If $r$ is proper, then $Y$ is $r$-tight if and only if there exists a constant $C$ and a superlinear function $f:[0, \infty) \longrightarrow \mathbb{R}$ such that

$$
\int_{X} f \circ r d \eta \leqslant C
$$

for all $\eta \in Y$. 


\section{References}

[1] R. A. Adams, Sobolev Spaces, Academic press, New York, 1975.

[2] L. Ambrosio, N. Gigli, and G. Savaré, Gradient flows, Gradient flows in metric spaces and in the space of probability measures, Lectures in Mathematics ETH Zürich. Birkhäuser Verlag, Basel, 2005.

[3] J. Ball, Convexity conditions and existence theorems in nonlinear elasticity, Arch. Rational Mech. Anal. 63 (1976/77), no. 4, 337-403.

[4] J. Ball and V. J. Mizel, One-dimensional variational problems whose minimizers do not satisfy the Euler-Lagrange equation, Arch. Rational Mech. Anal. 90 (1985), no. 4, 325-388.

[5] H. Berliocchi and J.-M. Lasry, Intégrandes normales et mesures paramétrées en calcul des variations, Bull. Soc. Math. France 101 (1973), 129-184.

[6] P. Bernard and B. Buffoni, Optimal mass transportation and Mather theory, J. Eur. Math. Soc. (JEMS) 9 (2007), no. 1, 85-121.

[7] P. Bernard, Young measures, superposition and transport, Indiana Univ. Math. J. 57 (2008), no. 1, 247-275.

[8] Y. Brenier, Minimal geodesics on groups of volume-preserving maps and generalized solutions of the Euler equations, Comm. Pure Appl. Math. 52 (1999), no. 4, 411-452.

[9] G. Buttazzo, Semicontinuity, Relaxation and Integral Representation in the Calculus of Variations, Pitman Research Notes in Mathematics Series, 207. Longman Scientific \& Technical, Harlow; copublished in the United States with John Wiley \& Sons, Inc., New York, 1989

[10] B. Dacorogna, Direct methods in the calculus of variations, Second edition. Applied Mathematical Sciences, 78. Springer, New York, 2008.

[11] L. C. Evans and R. F. Gariepy, Measure Theory and Fine Properties of Functions, Studies in Advanced Mathematics. CRC Press, Boca Raton, FL, 1992.

[12] M. Giaquinta, G. Modica, and J. Soucek, Cartesian Currents in the Calculus of Variations I, Springer, Berlin, 1998.

[13] _ Cartesian Currents in the Calculus of Variations II, Springer, Berlin, 1998.

[14] P. Pedregal, Parametrized Measures and Variational Principles, Progress in Nonlinear Differential Equations and their Applications, 30. Birkhäuser Verlag, Basel, 1997.

[15] M. Valadier, A course on Young measures, Rend. Istit. Mat. Univ. Trieste 24 (1994), 349-394.

[16] C. Villani, Topics in Optimal Transportation, Graduate Studies in Mathematics, 58. American Mathematical Society, Providence, RI, 2003.

[17] L. C. Young, Lectures on the Calculus of Variations and Optimal Control Theory, Second edition, Chelsea, 1980.

PATRICK BERNARD

Université PARIS-DAuphine

Ceremade, Umr Cnrs 7534

Pl. Du Maréchal de Lattre de Tassigny

75775 Paris Cedex 16, France

E-mail address: patrick.bernard@ceremade.dauphine.fr

Ugo Bessi

UNIVERSITÀ DI ROMA 3

Dipartimento di MATEMATiCA

Largo S. Leonardo Murialdo 1

00146 Roma, ItAlia

E-mail address: bessi@matrm3.mat.uniroma3.it 